\title{
Baixa adesão ao esquema vacinal anti-HPV por crianças e adolescentes
}

\section{Low adherence to the anti-HPV vaccine schedule by children and adolescentes}

\section{Baja adherencia a la vacunación contra el VPH entre niños y adolescentes}

Recebido: 13/10/2020

Aprovado: 06/03/2021

Publicado: 14/10/2021

\section{Jaqueline Fernandes Oliveira Pereira ${ }^{1}$ Qeren Hapuk Rodrigues Ferreira Fernandes ${ }^{2}$ Rita Terezinha de Oliveira Carneiro ${ }^{3}$}

Estudo descritivo e quantitativo realizado em 2018 na cidade de Sapeaçu, Bahia, com objetivo de identificar fatores associados à baixa adesão ao esquema vacinal anti-HPV por crianças e adolescentes. Foi aplicado questionário em 3 escolas. Os dados foram analisados pelos testes do qui-quadrado e backward. Participaram 119 estudantes: 53\% sexo feminino, 55\% pardos, $42 \%$ católicos, $46 \%$ renda de até um salário-mínimo e 61\% receberam vacina anti-HPV. As variáveis independentes foram: faixa etária $(p=0,89)$, raça/cor $(p=0,32)$, religião $(p=0,93)$, renda familiar $(p=0,60)$, entendimento do aluno sobre HPV $(p=0,16)$ e sobre o esquema vacinal anti-HPV $(p=0,976)$, ações da igreja que frequentam $(p=0,66)$, ações da escola $(p=0,657)$ e a filiação à rede de ensino da escola frequentada $(p=0,07)$, sexo dos estudantes $(p=0,002)$ e entendimento dos pais sobre o HPV $(p=0,012)$. Sexo dos participantes e o conhecimento dos seus pais sobre HPV foram fatores principais para baixa adesão ao esquema vacinal. Verificou-se a necessidade da elaboração de campanhas de sensibilização sobre a importância da vacinação anti-HPV voltadas para os pais e responsáveis, haja vista que são eles que atuam incisivamente na decisão de aderir ao esquema vacinal anti-HPV dos filhos.

Descritores: Vacinação; Doenças sexualmente transmissíveis; Neoplasias uterinas.

Descriptive and quantitative study carried out in 2018 in the city of Sapeaçu, in the state Bahia. It aimed to identify factors associated with low adherence to the anti-HPV vaccine regimen by children and adolescents. A questionnaire was applied in 3 schools. Data were analyzed using the chi-square and backward tests. 119 students participated: 53\% female, 55\% mixed-raced, 42\% Catholic, 46\% family income of up to one minimum wage and $61 \%$ received anti-HPV vaccine. The independent variables were: age group $(p=0.89)$, race/color $(p=0.32)$, religion $(p=0.93)$, family income $(p=0.60)$, student's understanding of HPV $(\mathrm{p}=0.16)$ and on the anti-HPV vaccine scheme $(\mathrm{p}=0.976)$, actions of the church they attend $(\mathrm{p}=0.66)$, school actions $(\mathrm{p}=0.657)$ and affiliation to the teaching network of school attended $(p=0.07)$, gender of students $(p=0.002)$ and parents' understanding of HPV $(p=0.012)$. Gender of the participants and their parents' knowledge about HPV were the main factors for low adherence to the vaccine schedule. There was a need to develop awareness campaigns on the importance of anti-HPV vaccination aimed at parents and guardians, given that they are the ones who act decisively in the decision to adhere to the anti-HPV vaccine schedule for their children.

Descriptors: Vaccination; Sexually transmitted diseases; Uterine neoplasms.

Estudo descritivo e quantitativo realizado em 2018 na cidade de Sapeaçu, Bahia, com objetivo de identificar fatores associados à baixa adesão ao esquema vacinal anti-HPV por crianças e adolescentes. Foi aplicado questionário em 3 escolas. Os dados foram analisados pelos testes do qui-quadrado e backward. Participaram 119 estudantes: 53\% sexo feminino, 55\% pardos, $42 \%$ católicos, $46 \%$ renda de até um salário-mínimo e 61\% receberam vacina anti-HPV. As variáveis independentes foram: faixa etária $(p=0,89)$, raça/cor $(p=0,32)$, religião $(p=0,93)$, renda familiar $(p=0,60)$, entendimento do aluno sobre HPV $(p=0,16)$ e sobre o esquema vacinal anti-HPV $(p=0,976)$, ações da igreja que frequentam $(p=0,66)$, ações da escola $(p=0,657)$ e a filiação à rede de ensino da escola frequentada $(p=0,07)$, sexo dos estudantes $(p=0,002)$ e entendimento dos pais sobre o HPV $(p=0,012)$. Sexo dos participantes e o conhecimento dos seus pais sobre HPV foram fatores principais para baixa adesão ao esquema vacinal. Verificou-se a necessidade da elaboração de campanhas de sensibilização sobre a importância da vacinação anti-HPV voltadas para os pais e responsáveis, haja vista que são eles que atuam incisivamente na decisão de aderir ao esquema vacinal anti-HPV dos filhos.

Descriptores: Vacunación; Enfermedades de transmisión sexual; Neoplasias uterinas.

1. Farmaceutica. Governador Mangabeira, BA, Brasil. ORCID: 0000-0003-0966-4121 E-mail: jakfop@outlook.com

2. Biotecnologista. Mestre em Biociências. Doutoranda em Biotecnologia em Saúde e Medicina Investigativa pelo Instituto Gonçalo Moniz Fiocruz, BA, Brasil. ORCID: 0000-0002-3172-9684 E-mail: qerenferreira@gmail.com

3. Bióloga. Especialista em Biologia Molecular. Mestre em Biotecnologia. Doutoranda em Biotecnologia em Saúde e Medicina Investigativa pelo Instituto Gonçalo Moniz - Fiocruz. Docente dos cursos de graduação em Biomedicina e Farmácia na Faculdade Maria Milza, Governador Mangabeira, BA, Brasil. ORCID: 0000-0002-7568-6487 E-mail: ritaterezinha@gmail.com 


\section{INTRODUÇÃO}

$\mathbf{V}$

acinação consiste em administrar uma solução inativada de material antigênico em indivíduos, visando o desenvolvimento de sua resposta imunológica frente a um determinado patógeno ${ }^{1}$. A vacinação de um grande número de pessoas numa dada população foi estimulada a partir do século XX e resultou na erradicação de diversas doenças infecciosas, o que evidencia sua eficácia. A Organização Mundial da Saúde (OMS) incentiva a prática de vacinação e faz um alerta sobre o despreparo mundial no enfretamento de surtos e reemergência de doenças infecciosas, devido à não adesão aos esquemas vacinais ${ }^{2}$.

HPV é uma infecção sexualmente transmissível (IST), causada pelo Papiloma vírus (Human papillomavirus) que apresenta tropismo na camada basal do endométrio, na qual se prolifera e desencadeia o processo infeccioso podendo evoluir para lesões características do câncer de colo uterino, que por sua vez é uma das principais causas de óbito de mulheres em todo o mundo ${ }^{3}$.

A infecção pelo HPV tem ocorrência mundial, contudo a alta carga da doença é registrada nos países em desenvolvimento. Ambos os sexos são susceptíveis, mas as mulheres mais afetadas são aquelas que se encontram em condições de maior vulnerabilidade socioeconômica ${ }^{4,5}$. No Brasil a taxa de infecção por HPV é mais alta na população jovem ${ }^{6}$.

Nos anos 1990 a Food and Drug Administration (FDA) autorizou a produção da vacina bivalente (Cervarix ${ }^{\circledR}$ ) que protege os indivíduos contra a infecção pelos sorotipos 16 e 18 do HPV, considerados os mais agressivos; e a vacina quadrivalente (Gardasil ${ }^{\circledR}$ ) que atua na defesa contra os sorotipos 1, 6, 16 e 18 do Papiloma vírus. Ambas as vacinas são distribuídas gratuitamente nas Unidades Básicas de Saúde (UBS) ${ }^{7}$.

0 esquema vacinal anti-HPV consiste na administração de duas doses da vacina com intervalo de 60 dias, e uma dose de reforço após 90 dias da administração da segunda dose ${ }^{8}$. Por recomendação da OMS a vacina anti-HPV deve ser oferecida às crianças ( 9 aos 11 anos) e adolescentes (12 aos 17 anos) para aumentar a prevenção contra o contágio pelo vírus ${ }^{7}$.

Campanhas de cunho educativo destinadas a popularizar e incentivar a vacinação antiHPV foram veiculadas nas mídias de grande alcance (televisão, rádio e internet) a partir de 2014, ano em que a vacina foi incorporada ao Programa Nacional de Imunização brasileiro ${ }^{7-9}$. Todavia, a adesão ao esquema vacinal contra o HPV no Brasil ainda é incipiente, em especial entre as crianças e os adolescentes. A cobertura vacinal para meninas dos nove aos 14 anos foi de $79,4 \%$ na primeira dose e 52,9\% na segunda dose no período entre 2013 a 2018 . Situação mais drástica é registrada entre os meninos de 11 a 14 anos, cuja cobertura é de 33\% na primeira dose, e apenas $8 \%$ para a segunda dose no mesmo período 7 .

Dentre as regiões brasileiras com alta prevalência de HPV destaca-se o Nordeste que registra a taxa de $58,1 \%$ de todos os casos absolutos notificados no Brasil nos últimos anos ${ }^{9}$. 0 Recôncavo baiano é uma extensa área territorial pertencente a região nordestina do país e caracterizada pela cultura sertaneja. Nessa região se encontram instalações de importantes centros de pesquisa e educação, configurando o lugar como um polo econômico e educativo ${ }^{10}$.

Em virtude da correlação entre o processo infeccioso pelo HPV e a ocorrência de câncer uterino, e levando em consideração a possibilidade de prevenir novos casos da infecção por meio da vacina anti-HPV é justificável a condução desse estudo, assim, o objetivo desse trabalho é identificar fatores associados à baixa adesão ao esquema vacinal anti-HPV por crianças e adolescentes.

\section{MÉTODO}

Trata-se de um estudo descritivo e quantitativo realizado com crianças com idades entre nove aos 11 anos e adolescentes com 11 a 14 anos devidamente matriculados em escolas dos Ensinos Fundamental e Médio na região do Recôncavo baiano. A participação dos estudantes foi condicionada à anuência dada por seus respectivos pais ou responsáveis. 
As escolas coparticipantes dessa pesquisa, nas quais os dados foram coletados, estão identificadas como A que se insere na rede particular de ensino e está localizada na área urbana da cidade de Sapeaçu, Bahia, Brasil; e as escolas B e C que ofertam educação gratuita aos estudantes e se localizam respectivamente nas áreas urbana e rural da mesma cidade.

Um formulário composto por 32 questões objetivas foi elaborado como instrumento de pesquisa e entregue aleatoriamente aos estudantes, em dias e horários previamente agendados com as respectivas direções e corpo docente.

As questões se distribuíam em cinco eixos: (i) o que é HPV e como ocorre a infecção; (ii) importância da vacina contra o processo infeccioso pelo HPV e sobre o esquema de imunização; (iii) fonte de informação sobre a doença e sua prevenção; (iv) conhecimento dos pais ou responsáveis sobre a infecção pelo HPV e as vias de prevenção da doença e $(v)$ caracterização socioeconômica dos participantes dessa pesquisa. A coleta de dados ocorreu entre os meses de setembro e outubro de 2018.

Os dados foram analisados por meio da comparação das respostas obtidas com as informações disponíveis no Guia Prático sobre o HPV3. As respostas foram categorizadas numa escala de 1 a 4 para classificação, a saber: excelente, boa, regular e ruim, respectivamente. Para as perguntas cujas respostas eram "sim/não" foi adotado o número 1 para sim, e o número 2 para não.

A soma das questões corretas foi utilizada na classificação dos perfis de conhecimento dos estudantes em: "EXCELENTE" para somatório com percentual $>50 \%$ de respostas corretas; "BOM" para somatório com percentual igual a 50\% das respostas corretas; "REGULAR" para somatório $\leq 50 \% \geq$ de respostas corretas e "RUIM" para percentual $<50 \%$ de respostas corretas. Posteriormente, as respostas foram tabuladas em planilha Excel (Microsoft ${ }^{\circledR}$ ) e submetidas ao teste de qui-quadrado $\left(\chi^{2}\right)$ para análises bivariada e multivariada, com exclusão progressiva das variáveis que apresentaram nível de significância superior a 0,05 utilizando a técnica de backward, no programa STATA (versão 7.2) adotando valor $\alpha$ de 5\%.

0 termo "aderência ao esquema vacinal" aqui foi empregado para caracterizar duas situações não excludentes entre elas: a) quando os participantes da pesquisa e seus pais apresentaram um perfil de conhecimento bom sobre a infecção e a prevenção do HPV e b) para os estudantes que receberam ao menos uma dose da vacina anti-HPV.

Esse trabalho foi aprovado pelo Comitê de Ética em Pesquisa da Faculdade Maria Milza (CEP-FAMAM) e registrada com parecer de número 97827018.1.0000.5025. Um ofício foi emitido em nome da Coordenação do Curso de Farmácia da FAMAM à direção de escolas solicitando a anuência para coleta dos dados.

Os pais ou responsáveis dos estudantes tomaram ciência dos objetivos, metodologia e eventuais riscos dessa pesquisa por meio de apresentação da proposta de trabalho durante reuniões semestral de "Pais e Mestres", realizadas entre os meses de julho e agosto de 2018. As referidas reuniões contaram com a participação das respectivas direções e corpo docente das escolas coparticipantes. Nessa ocasião foram entregues o Termo de Consentimento Livre Esclarecido (TCLE) para os pais e responsáveis, enquanto as crianças e adolescentes receberam o Termo de Assentimento.

\section{RESULTADOS}

No período, identificou-se 617 estudantes com idade entre 9 a 14 anos matriculados nas três escolas coparticipantes. Contudo, a amostra foi constituída por 119 participantes ( $\mathrm{n}=617$, 17\%). A estratificação dos participantes por sexo revelou predominância de meninas (53\%), e não foi verificada evasão escolar ou distorção idade-série nas escolas das redes pública e privada de ensino.

Os dados revelaram que sobre o ciclo infeccioso causado pelo HPV e a importância da vacina anti-HPV 45,3\% apresentaram conhecimento excelente; 3,4\% conhecimento bom; $27 \%$ 
conhecimento regular; e, $24,3 \%$ conhecimento ruim. Ao mesmo tempo, $61 \%$ declararam ter recebido ao menos uma dose da vacina anti-HPV.

Estudantes da escola A obtiveram um percentual $45 \%$ de todas as respostas consideradas como excelente, e os estudantes das escolas B e C apresentaram respectivamente os percentuais de $34 \%$ e aproximadamente $29 \%$ dos melhores índices de conhecimento sobre o tema.

A Tabela 1 descreve os dados que caracterizam a amostra analisada em seus aspectos sociais e demográficos.

Tabela 1. Estudantes quanto a dados sociodemográficos predominantes conforme escolas. Sapeaçu, Bahia. 2018.

\begin{tabular}{llll}
\hline Variáveis & Escola A & Escola B & Escola C \\
\hline Idade (anos) & 10 e 14 & 12 e 13 & 10 \\
Sexo & Feminino (52\%) & Feminino (61\%) & Masculino (54\%) \\
Raça/cor & Parda (41,5\%) & Parda (46\%) & Parda (50\%) \\
Religião & Católica (54\%) & Nenhuma (50\%) & Católica (50\%) \\
Renda familiar & Superior a um salário-mínimo (48\%) & Menor que um salário-mínimo (50\%) & Até um salário-mínimo (54\%) \\
\hline
\end{tabular}

O perfil de conhecimento dos pais ou responsáveis sobre o processo infeccioso pelo HPV e a importância da vacina anti-HPV foi constituído por $11 \%$ que apresentaram conhecimento excelente; $31 \%$ com bom entendimento; $18 \%$ apresentaram conhecimento regular e 33\% com conhecimento ruim. Em 8,3\% dos formulários foram devolvidos sem registros do conhecimento dos pais.

Internet e televisão foram citados como as principais fontes de informação sobre vias de contaminação e sobre as formas de prevenção contra o HPV. As instituições que mais contribuem na divulgação de informações sobre a doença bem como sobre a vacinação são as escolas e as unidades de saúde.

Análise de estatística descritiva indicou que os fatores de maior influência para a baixa adesão ao esquema vacinal anti-HPV foram: sexo do estudante $(p=0,003)$ e o conhecimento dos pais ou responsáveis sobre o assunto $(p=0,003)$. Os demais determinantes sociodemográficos analisados, como: renda familiar, raça/cor, idade ou religião não tiveram diferença significativa (Tabela 2).

0 modelo teórico da análise multivariada adotou a adesão ao esquema vacinal como variável dependente. As variáveis independentes foram retiradas do modelo de forma sequencial por: faixa etária $(p=0,891)$, raça/cor $(p=0,325)$, religião $(p=0,939)$, renda familiar $(p=0,60)$, entendimento do aluno sobre o processo infeccioso pelo $\operatorname{HPV}(p=0,167)$, entendimento do aluno sobre o esquema vacinal contra o HPV $(p=0,976)$, ações da igreja que os estudantes frequentam $(p=0,66)$, ações das escolas nas quais estão respectivamente matriculados $(p=0,657)$ e a filiação da escola a rede de ensino, i.e.: particular ou pública $(p=0,07)$.

Duas variáveis independentes apresentaram associação na análise multivariada: sexo dos estudantes (OR=3,125; IC95\%: 1,5-6,49; $p=0,002$ ) e o entendimento dos pais ou responsáveis sobre a infecção pelo HPV (OR=2,81; IC95\%: 1,25-6,30; $p=0,012)$, confirmando a análise bivariada. 
Tabela 2. Estudantes segundo variáveis independentes analisadas sobre a temática conhecimento acerca do HPV e vacinação. Sapeaçu, Bahia. 2018.

\begin{tabular}{|c|c|c|c|c|}
\hline Variáveis & $\begin{array}{l}\text { Adesão ao esquema vacinal anti-HPV } \\
(n=73 / 119)\end{array}$ & $\begin{array}{c}\text { Não adesão ao esquema vacinal } \\
\text { anti-HPV }(n=46 / 119)\end{array}$ & Total $(n=119)$ & $\begin{array}{l}p \text {-valor } \\
(\alpha 0,05)\end{array}$ \\
\hline & $\%$ & $\%$ & $\%$ & \\
\hline Raça/cor & & & & 0,729 \\
\hline Negro & 30,1 & 36,9 & 32,7 & \\
\hline Mestiço & 8,2 & 4,3 & 6,7 & \\
\hline Branco & 15,1 & 17,4 & 15,9 & \\
\hline Pardo & 46,6 & 41,3 & 44,5 & \\
\hline Idade & & & & 0,593 \\
\hline 09 anos & 4,1 & 2,1 & 3,4 & \\
\hline 10 anos & 27,4 & 28,2 & 27,7 & \\
\hline 11 anos & 20,5 & 19,5 & 20,1 & \\
\hline 12 anos & 9,5 & 21,7 & 14,2 & \\
\hline 13 anos & 19,2 & 13,0 & 16,8 & \\
\hline 14 anos & 19,2 & 15,2 & 17,6 & \\
\hline Sexo & & & & 0,000 \\
\hline Feminino & 65,7 & 32,6 & 52,9 & \\
\hline Masculino & 34,2 & 67,4 & 47,1 & \\
\hline Religião & & & & 0,524 \\
\hline Católico & 52,0 & 50,0 & 51,3 & \\
\hline Protestante & 15,1 & 10,9 & 13,4 & \\
\hline Espírita & 2,7 & 0,0 & 1,7 & \\
\hline Matrizes Africana & 1,4 & 0,0 & 0,9 & \\
\hline Nenhuma & 28,7 & 39,1 & 32,7 & \\
\hline Ações realizadas pelas escolas & & & & 0,259 \\
\hline Sim & 38,4 & 28,3 & 34,4 & \\
\hline Não & 61,6 & 71,7 & 65,5 & \\
\hline Ações realizadas pelas Unidades de Saúde & & & & 0,657 \\
\hline Sim & 62,6 & 58,3 & 61,3 & \\
\hline Não & 37,4 & 41,7 & 38,7 & \\
\hline
\end{tabular}


Tabela 2. Estudantes segundo variáveis independentes analisadas sobre a temática conhecimento acerca do HPV e vacinação. Sapeaçu, Bahia. 2018. [Continuação]

\begin{tabular}{|c|c|c|c|c|}
\hline Variáveis & $\begin{array}{c}\text { Adesão ao esquema vacinal } \\
\text { anti-HPV }(n=73 / 119)\end{array}$ & $\begin{array}{c}\text { Não adesão ao esquema } \\
\text { vacinal anti-HPV }(n=46 / 119)\end{array}$ & Total $(n=119)$ & $\begin{array}{l}p \text {-valor } \\
(\alpha 0,05)\end{array}$ \\
\hline & $\%$ & $\%$ & $\%$ & \\
\hline Ações promovidas pelas igrejas que frequentam & & & & 0,172 \\
\hline Sim & 19,2 & 10,9 & 15,9 & \\
\hline Não & 12,3 & 23,9 & 16,8 & \\
\hline Não se aplica & 68,5 & 65,2 & 67,2 & \\
\hline Conhecimento dos participantes sobre HPV & & & & 0,959 \\
\hline Excelente & 43,8 & 45,6 & 44,5 & \\
\hline Bom & 2,7 & 4,3 & 3,4 & \\
\hline Regular & 27,4 & 26,1 & 26,9 & \\
\hline Ruim & 26,0 & 23,9 & 25,2 & \\
\hline $\begin{array}{l}\text { Conhecimento dos pais e responsáveis sobre a vacina } \\
\text { anti-HPV }\end{array}$ & & & & 0,003 \\
\hline Excelente & 19,8 & 6,5 & 14,3 & \\
\hline Bom & 43,8 & 28,3 & 37,8 & \\
\hline Regular & 16,4 & 13,0 & 15,1 & \\
\hline Ruim & 20,5 & 52,2 & 32,8 & \\
\hline Renda familiar & & & & 0,647 \\
\hline Menos de um salário-mínimo & 15,1 & 21,7 & 17,7 & \\
\hline Até um salário-mínimo & 47,9 & 43,5 & 46,2 & \\
\hline Mais de um salário-mínimo & 37,0 & 34,8 & 36,1 & \\
\hline Conhecimento dos participantes sobre a vacina & & & & 0,278 \\
\hline Excelente & 4,1 & 4,3 & 4,2 & \\
\hline Bom & 17,8 & 10,8 & 15,1 & \\
\hline Regular & 32,9 & 21,7 & 28,6 & \\
\hline Ruim & 45,2 & 63,0 & 52,1 & \\
\hline
\end{tabular}




\section{DISCUSSÃO}

Participantes do sexo feminino apresentam maior conhecimento e aceitabilidade da vacina anti-HPV em relação aos estudantes do sexo masculino. Um fato que remete a dados históricos e indica que as mulheres se mostram mais preocupadas em se prevenir de doenças ${ }^{11}$. Em tempo, ressalva-se que os percentuais de estudantes dos sexos masculino e feminino nas escolas visitadas são similares entre si, portanto o maior percentual de meninas não é fruto de viés de seleção do público feminino na condução desse estudo.

Os estudantes da escola privada apresentaram entendimento melhor sobre o ciclo infeccioso e a importância da vacina anti-HPV em comparação aos participantes matriculados em ambas as escolas públicas aqui analisadas. Essa diferença pode estar associada ao fato de que os estudantes das escolas privadas possuem condição econômica que lhes proporciona melhor acesso à informação por meio de livros e internet o que facilita seu conhecimento, como relatado em trabalho anterior ${ }^{12}$.

Em contrapartida, os estudantes das escolas públicas, em sua maioria, apresentam renda familiar igual a um salário-mínimo, o que indiretamente revela o baixo índice de escolaridade dos seus familiares de modo a influenciar fortemente em decisões importantes sobre suas vidas $^{13}$, como na decisão em não aderir ao programa de prevenção contra o HPV.

Indivíduos adeptos a religiões mais conservadoras geralmente associam a vacinação antiHPV com o início da atividade sexual pelos adolescentes, e por esse motivo preferem se abster da imunização para seus filhos ${ }^{4,14}$. Todavia, no presente estudo a maioria dos participantes se declararam católicos, e a análise estatística descritiva revelou que esse fator não exerceu influência significativa para a baixa adesão ao esquema vacinal anti-HPV.

0 conhecimento dos pais e responsáveis é fator determinante na adesão ao esquema vacinal anti-HPV. Dados que corroboram com os resultados de estudos realizados no Brasil e outros países evidenciaram que a baixa cobertura vacinal de crianças e adolescentes ocorre em virtude da não aceitação da vacina por parte dos pais e responsáveis ${ }^{15-18}$.

A decisão dos pais e responsáveis na condução de seus filhos ou tutelados para serem vacinados é influenciada pelos meios de comunicação empregados para difusão do conhecimento sobre o tema. Esse contexto colabora para o entendimento sobre o impacto que as mídias exercem na promoção de campanhas de saúde ${ }^{14}$. A metodologia aqui empregada não contempla a investigação sobre o tipo e a qualidade do acesso das informações sobre o HPV acessados pelos estudantes e seus pais ou responsáveis. Porém, foi observado que o acesso à internet por meio do celular é algo comum, ao menos pelos estudantes. Esse contexto revela a importância e urgência de campanhas informativas voltadas para crianças e adolescentes, mas também para os pais e responsáveis ${ }^{19}$.

Apesar da contribuição atribuída aos meios de comunicação na promoção da saúde, vale ressaltar que as informações precisam ser claras e fidedignas. Caso contrário, a difusão de informações de maneira errônea pode ocorrer e, consequentemente, gerar um impacto negativo na adesão de programas de controle e prevenção de doenças ${ }^{14-20}$. Entre os anos de 2014 e 2017 foi registrada uma agitação mundial em prol do movimento anti-vacina, apontada como responsável para aumento de $87 \%$ de não aceitação de vacinas para as mais variadas doenças somente nesse período ${ }^{2}$.

Os estudantes pesquisados receberam ao menos uma dose da vacina. Porém, é importante salientar que a imunização contra o HPV só é efetiva quando o esquema vacinal é completado, ou seja, quando duas doses da vacina e a dose de reforço são administradas ${ }^{8}$.

O município de Sapeaçu, Bahia dispõe de oito unidades de saúde equipadas com sala de vacina e técnico habilitado para sua aplicação. Entretanto, dados não publicados e cedidos pela Secretaria Municipal de Saúde (SMS) revelaram que a adesão ao esquema vacinal anti-HPV foi menor que 35\% no município, percentual abaixo da taxa nacional no período desse estudo. Foram observados registros de visitas domiciliares realizadas pelos Agentes Comunitários de Saúde (ACS) com intuito de difundir informações corretas e incentivar a adesão aos programas 
de vacinação. Além disso, foi verificada a parceria entre UBS e as escolas do município por meio do Programa Saúde na Escola (PSE), criado pelo Governo Federal com vistas a realizar palestras e oficinas voltadas para a promoção de atividades voltadas para saúde e bem-estar da população.

O Ministério da Saúde disponibiliza gratuitamente cartilhas sobre o HPV2, e entre 2014 a 2016 promoveu campanhas de sensibilização em diversos meios de comunicação com uma mensagem voltada ao público jovem. Contudo, se percebe a necessidade da elaboração de campanhas de sensibilização sobre a importância da vacinação anti-HPV voltadas para os pais e responsáveis, haja vista que são eles os principais agentes na decisão em aderir ao esquema vacinal anti-HPV dos seus filhos e tutelados menores de idade.

\section{CONCLUSÃo}

0 presente estudo elucidou que o sexo dos participantes desse estudo e o conhecimento de seus pais e responsáveis são os fatores que interferem de modo significativo para a não adesão à vacina anti-HPV na cidade de Sapeaçu, Bahia. Sugere-se assim, a elaboração das campanhas e programas de incentivo à vacinação anti-HPV voltados para os pais e responsáveis, bem como o fortalecimento de parcerias entre escolas, unidades de saúde e igrejas na promoção de ações dessa natureza com vista a incentivar a adesão de crianças e adolescentes do sexo masculino.

Esse estudo apresentou como limitação o fato de não poder considerar uma parcela dos formulários entregues pelas crianças e adolescentes que mostraram interesse em participar da pesquisa, mas que em contrapartida não apresentaram a anuência dos seus respectivos pais ou responsáveis, concedida pela assinatura do TCLE. Esse dado indiretamente reforça nossos achados a respeito da incompreensão por partes dos pais e responsáveis sobre a importância de aderir ao programa de imunização contra o HPV.

0 presente estudo alerta sobre a necessidade de sensibilizar pais e responsáveis para um problema de saúde passível de tratamento eficaz, mas que pode evoluir para câncer agressivo e de alta mortalidade.

\section{REFERÊNCIAS}

1. Kocourkova A, Honegr J, Kuca K, Danova J. Vaccine ingredientes: Components that influence vaccine eficacy. Mini Rev Med Chem. [Internet]. 2017 [citado em 05 maio 2019]; 17(5):45166. Disponível em: https://pubmed.ncbi.nlm.nih.gov/27488583/. DOI: 10.2174/ 1389557516666160801103303

2. Marshal GS. Vaccine hesitancy, history, and human nature: the 2018 Stanley A. Plotkin Lecture. J Pediatric Infect Dis Soc. [Internet]. 2019 [citado em 28 mar 2019]; 8(1):18. Disponível em: https://pubmed.ncbi.nlm.nih.gov/33513237/. DOI: 10.1093/jpids/piy082

3. Ministério da Saúde (Brasil), Departamento de Vigilância de Doenças Transmissíveis. Guia Prático sobre HPV: perguntas e respostas [Internet]. Brasília, DF: [Ministério da Saúde]; 2017 [citado em 05 maio 2019]. 45p. Disponível em: https://antigo.saude.gov.br/images/pdf/2017/dezembro/07/Perguntas-e-respostas-HPV.pdf

4. Mollers M, Lubbers K, Spoelstra SK, Weijmar-Schultz WC, Daemen T, Westra TA, et al. Equity in human papilloma virus vaccination uptake?: sexual behaviour, knowledge and demographics in a cross-sectional study in (un) vaccinated girls in the Netherlands. BMC Public Health [Internet]. 2014 [citado em 28 maio 2019]; 14(1):288. Disponível em: https://bmcpublichealth.biomedcentral.com/articles/10.1186/1471-2458-14-288. $\quad$ DOI: 10.1186/1471-2458-14-288

5. Von Karsa L, Suonio E, Lignini T, Ducarroz S, Anttila A, editors. Current status and future directions of breast and cervical cancer prevention and early detection in Belarus. Report of Expert Mission to Minsk, Belarus, 15-18 February 2011 [Internet]. Lyon, France: IARC/WHO; 
2012 [citado em 28 abr 2019]. 155p. Disponível em: http://www.iarc.fr/en/publications/pdfs-online/wrk/wrk6/Belarus_Report.pdf

6. Ministério da Saúde (Brasil), Secretaria de Vigilância em Saúde, Departamento de Vigilância das Doenças Transmissíveis, Coordenação-Geral do Programa Nacional de Imunizações. Informe técnico da ampliação da oferta das vacinas papilomavírus humano 6, 11, 16 e 18 (recombinante) - vacina HPV quadrivalente e meningocócica C (conjugada) [Internet]. Brasília, DF: [Ministério da Saúde]; 2018 [citado em 05 maio 2019]. 39p. Disponível em: https://portalarquivos2.saude.gov.br/images/pdf/2018/marco/14/Informe-T--cnico-HPVMENINGITE.pdf

7. Lobão WM, Duarte FG, Burns JD, Santos CAST, Almeida MCC, Reingold A, et al. Low coverage of HPV vaccination in the national immunization programme in Brazil: parental vaccine refusal or barriers in healthservice based vaccine delivery? Plos One [Internet]. 2018 [citado em 28 maio 2019]; 13(11):1-14. Disponível em: https://www.ncbi.nlm.nih.gov/pmc/articles/PMC6231618/pdf/pone.0206726.pdf. $\quad$ DOI: 10.1371/journal.pone.0206726.eCollection2018

8. Zardo GP, Farah FP, Mendes FG, Franco CAG, Molina GVM, Melo GN, et al. Vacina como agente de imunização contra o HPV. Ciênc Saúde Colet. [Internet]. 2014; [citado em 28 maio 2019]; 19(9):3799-808. Disponível em:

https://www.scielo.br/j/csc/a/vhx9ghBGgKKWCL6CXJ69X7N/?lang=pt\&format=pdf. $\quad$ DOI: 10.1590/1413-81232014199.01532013

9. Osis MJD, Duarte GA, Sousa MH. Conhecimento e atitude de usuários do SUS sobre o HPV e as vacinas disponíveis no Brasil. Rev Saúde Pública [Internet]. 2014 [citado em 28 maio 2019]; 48(1):123-33. Disponível em: https://scielosp.org/pdf/rsp/2014.v48n1/123-133/pt. DOI: https://doi.org/10.1590/S0034-8910.2014048005026

10. Alban M. O novo enigma baiano, a questão urbana-regional e a alternativa de uma nova capital [Internet]. In: Anais do XI Encontro Nacional da Associação Nacional de Pós-Graduação e Pesquisa em Planejamento Urbano e Regional - ANPUR; 2005; Salvador. São Paulo: ANPUR; 2005 [citado em 11 ago 2021]. Disponível em: http://anais.anpur.org.br/index.php/anaisenanpur/issue/archive

11. Kreuger MRO, Lizott LS, Friedrich HA. Imunização contra HPV: nível de conhecimento dos adolescentes. Adolesc Saúde [Internet]. 2017 [citado em 28 maio 2019]; 14(3):3845. Disponível em: http://adolescenciaesaude.com/app_offline.htm

12. Peixoto AMCL, Valença PAM, Amorim VCSA. Conhecimento, atitudes e práticas de adolescentes e pais sobre imunização na adolescência: revisão sistemática. Rev Bras Promoç Saúde [Internet]. 2018 [citado em 28 maio 2019]; 31(3):1-10. Disponível em: https://periodicos.unifor.br/RBPS/article/view/7805. DOI: 10.5020/18061230.2018.7805

13. Dias JMG, Fontes TSMO, Araújo DM, Passos AMPR. Conhecimento sobre a vacina contra o papilomavírus humano (HPV) entre adolescentes de escolas públicas e particulares de Aracaju. Adolesc Saúde [Internet]. 2016 [citado em 28 maio 2019]; 13(2):77-88. Disponível em: http://adolescenciaesaude.com/app_offline.htm

14. Quevedo JP, Inacio M, Wieczorkievicz AM, Invernizzi N. A política de vacinação contra o HPV no Brasil: a comunicação pública oficial e midiática face à emergência de controvérsias. Rev Tecnol Soc. [Internet] 2016 [citado em 28 maio 2019]; 12(24):1-26. Disponível em: https://periodicos.utfpr.edu.br/rts/article/view/3206/2622. DOI: 10.3895/rts.v12n24.3206 15. Carpiano RM, Polonijo NA, Gilbert N, Cantin L, Dubé E. Socioeconomic status differences in parental immunization attitudes and child immunization in Canada: findings from the 2013 Childhood National Immunization Coverage Survey (CNICS). Prev Med. [Internet]. 2019 [citado em 28 out 2019]; 123(2019):278-87. Disponível em: https://www.sciencedirect.com/science/article/abs/pii/S0091743519301094?via\%3Dihub. DOI: https://doi.org/10.1016/j.ypmed.2019.03.033 
16. Kornides ML, McRee AL, Gilkey, MB. Parents who decline HPV vaccination: who later accepts and why? Acad Pediatr. [Internet]. 2018 [citado em 28 out 2019]; 18(2S):S37-S43. Disponível

em: https://www.ncbi.nlm.nih.gov/pmc/articles/PMC5859546/pdf/nihms949872.pdf. $\quad$ DOI: 10.1016/j.acap.2017.06.008

17. Gilbert NL, Gilmour H, Dubé E, Wilson SE, Laroche J. Estimates and determinants of HPV non-vaccination and vaccine refusal in girls 12 to $14 \mathrm{y}$ of age in Canada: results from the Childhood National Immunization Coverage Survey. 2013. Hum Vaccin Immunotherapeutics [Internet]. 2016 [citado em 28 out 2019]; 12(6):1484-90. Disponível em: $\quad$ https://www.ncbi.nlm.nih.gov/pmc/articles/PMC4964714/pdf/khvi-12-061153207.pdf. DOI: 10.1080 / 21645515.2016.1153207

18. Notejane M, Zunino C, Méndez P, García L, Pérez W. Estado vacunal y motivos de no vacunación contra el virus del papiloma humano en adolescentes admitidas en el Hospital Pediátrico del Centro Hospitalario Pereira Rossell. Rev Méd Urug. [Internet] 2018 [citado em 28 out 2019]; 34(2):76-81. Disponível em: http://www.scielo.edu.uy/pdf/rmu/v34n2/16880390-rmu-34-02-10.pdf

19. Restivo V, Constantino C, Fazio TF, Casuccio N, D'Angelo C, Vitale F, et al. Factors associated with HPV vaccine refusal among young adult women after ten years of vaccine implementation. Int J Environ Res Public Health [Internet]. 2018 [citado em 28 out 2019]; 15(4):770. Disponível em: https://www.ncbi.nlm.nih.gov/pmc/articles/PMC5923812/. DOI: 10.3390/ijerph15040770

20. Silva KB, Bezerra AFB, Chaves LDP, Tanaka OY. Integralidade no cuidado ao câncer do colo do útero: avaliação do acesso. Rev Saúde Pública [Internet]. 2014 [citado em 28 out 2019]; 48(2):240-8. Disponível em: https://scielosp.org/pdf/rsp/2014.v48n2/240-248/pt. DOI: https://doi.org/10.1590/S0034-8910.2014048004852

Editora Associada: Vania Del Arco Paschoal

\section{CONTRIBUIÇÕES}

Jaqueline Fernandes Oliveira Pereira contribuiu na coleta e análise dos dados e redação. Qeren Hapuk Rodrigues Ferreira Fernandes colaborou na análise dos dados. Rita Terezinha de Oliveira Carneiro atuou na concepção, coleta e análise dos dados, redação e revisão.

\section{Como citar este artigo (Vancouver)}

Pereira, JFO, Fernandes QHRF, Carneiro RTO. Baixa adesão ao esquema vacinal anti-HPV por crianças e adolescentes. REFACS [Internet]. 2021 [citado em inserir dia, mês e ano de acesso]; 9(4):870-79. Disponível em: inserir link de acesso. DOI: inserir link do DOI.

\section{Como citar este artigo (ABNT)}

PEREIRA, J. F. O.; FERNANDES, Q. H. R. F.; CARNEIRO, R. T. de O. Baixa adesão ao esquema vacinal anti-HPV por crianças e adolescentes. REFACS, Uberaba, MG, v. 9, n. 4, p. 870-79, 2021. Disponível em: inserir link de acesso. Acesso em: inserir dia, mês e ano de acesso. DOI: inserir link do DOI.

\section{Como citar este artigo (APA)}

Pereira, J.F.O., Fernandes, Q.H.R.F., \& Carneiro, R.T.O. (2021). Baixa adesão ao esquema vacinal antiHPV por crianças e adolescentes. REFACS, 9(4), 870-79. Recuperado em: inserir dia, mês e ano de acesso de inserir link de acesso. DOI: inserir link do DOI. 